\title{
Caractérisation structurale des formations naturelles enrichies en essences forestières locales: cas des vertisols de la Lama (Benin)
}

\author{
Laurent DJODJOUWIN *, Romain GLELE KAKAÏ et Brice SINSIN \\ Laboratoire d'Ecologie Appliquée, Faculté des Sciences Agronomiques, Université d'Abomey-Calavi, 01 BP \\ 526 Cotonou, Bénin. \\ *Auteur correspondant, E-mail :djodjou@yahoo.fr; Tél. +22995 509104.
}

\section{RESUME}

Trois espèces à savoir, Afzelia africana Sm., Khaya grandifoliola Welw C.D.C. et Khaya senegalensis Desr. introduites pour l'enrichissement des zones dégradées de la forêt classée de la Lama, ont été considérées afin d'évaluer l'impact de leur introduction sur la structure et l'écologie de ces zones. Un total de 24 placeaux, tous distants d'au moins $100 \mathrm{~m}$ l'un de l'autre ont été installés dans les plantations d'enrichissement de 17, 18, 19 et 20 ans d'âge. Dans ces placeaux, tous les arbres de diamètre 1, $30 \mathrm{~m}$ du sol (dbh) supérieur ou égal à 10 $\mathrm{cm}$ ont été mesurés. Par ailleurs, la hauteur totale de tous les sujets de chacune des trois espèces cibles a été mesurée. Ces données ont également servi à modéliser la relation entre le diamètre et la hauteur. L'analyse de la covariance a été réalisée pour comparer les peuplements enrichis et les populations des trois espèces sur la base des paramètres dendrométriques en considérant successivement comme covariable, l'âge et l'écartement des plantations. Par contre, les comparaisons basées sur la densité ont été faites par le test de Kruskal-Wallis. La densité moyenne des trois espèces (291 sujets/ha) représente $40 \%$ de la densité globale des peuplements enrichis avec une contribution de $20 \%$ environ $\left(5,9 \mathrm{~m}^{2} / \mathrm{ha}\right)$ à la surface terrière globale de ces peuplements. Quel que soit l'écartement, les structures en diamètres sont dissymétriques avec la prédominance des petits sujets. Ce qui est en partie dû à la compétition qu'exercent les espèces naturelles préexistantes sur les espèces introduites. Au vu des caractéristiques écologiques, dendrométriques et structurales obtenues, il est nécessaire de réaliser des éclaircies destinées à favoriser la croissance et le dévéloppement des sujets d'avenir.

(C) 2011 International Formulae Group. All rights reserved.

Mots clés : Plantations d'enrichissement, zones dégradées, essences introduites, vertisol, Bénin.

\section{INTRODUCTION}

Le reboisement des surfaces dégradées est un moyen efficace et rapide pour reproduire le peuplement et satisfaire les besoins en bois (Dupuy, 1989). Afin d'assurer la pérennité des ressources et le maintien de la couverture forestière, l'une des solutions envisageables à court terme consiste à identifier et à introduire des espèces adaptées (Dupuy, 1992). L'approche devrait donc permettre d'augmenter la production ligneuse sans perturber les formations naturelles préexistantes.

Le Noyau Central de la forêt classée de la Lama au Bénin, donne un intérêt pour des peuplements complexes (peuplements irréguliers, mélange d'espèces, structure hétérogène) supposés mieux répondre aux fonctions de protection, de production (Ginisty, 2008) et d'accueil du public (Barthod, 1996). L'hétérogénéité de ces peuplements constitue une source de diversité 
d'habitats notamment, favorable aux espèces animales et végétales.

Les plantations d'enrichissement de la forêt classée de la Lama constituent l'un des tous premiers (depuis 1989) essais d'enrichissement de forêts dégradées au Bénin (Djodjouwin et Sinsin, 2007). Elles ont été établies en suivant différents modes d'enrichissement dans l'espoir d'un rétablissement écologique de la forêt dans ses zones dégradées. Parmi une vingtaine d'essences introduites, trois ont été choisies dans la présente étude au regard, non seulement, de leurs usages multiples, mais aussi de leur statut d'espèces menacées de disparition. Il s'agit de Afzelia africana Sm, Khaya grandifoliola Welw C.D.C et Khaya senegalensis Desr. Vingt ans après cet enrichissement, il est nécessaire d'en analyser l'impact sur la structure et l'écologie de ces zones dégradées d'une part, et sur le développement des espèces introduites, d'autre part. L'objectif principal de la présente étude est de contribuer à l'évaluation des plantations d'enrichissement de formations forestières naturelles dégradées à travers une analyse structurale des stations enrichies en $A$. africana, $K$. grandifoliola et $K$. senegalensis et l'analyse des effets de compétition sur la croissance des arbres de ces trois espèces.

\section{MATERIEL ET METHODES \\ Milieu d'étude}

La forêt classée de la Lama est située au sud du Bénin et s'étend entre $6^{\circ} 55^{\prime}$ et 7 ${ }^{\circ} 00^{\prime}$ de latitude Nord et entre $2{ }^{\circ} 4{ }^{\prime}$ et $2^{\circ} 12^{\prime}$ de longitude Est. Sa superficie totale est estimée à 16250 ha dont environ 11000 ha de massif forestier. Actuellement, la zone centrée sur les vestiges de la forêt naturelle, dénommée Noyau Central couvre une superficie de 4777 ha dont 292 ha de plantations de Tectona grandis et de Gmelina arborea et 1900 ha de forêt dense, le reste étant composé de forêts dégradées et de jachères enrichies (Adomou et al., 2007). La pluviométrie moyenne annuelle de la zone oscille entre $1000 \mathrm{~mm}$ et $1200 \mathrm{~mm}$. La température moyenne varie entre 25 et 29 ${ }^{\circ} \mathrm{C}$ et l'humidité relative, entre 69 et $97 \%$. Les sols de la forêt classée de la Lama sont des vertisols de texture argilo-sableuse (Specht, 2002 ; Sinsin et al., 2003) comportant une forte teneur en matière organique sur une profondeur de 1,0 m à 1,2 $\mathrm{m}$. Ce sont des sols fertiles mais qui, en raison de leurs propriétés physiques, sont difficiles à cultiver.

Pour les besoins de l'étude, la carte de végétation de la forêt classée de la Lama et d'autres documents relatifs aux plantations d'enrichissement ont été utilisés pour retenir les sites d'inventaire où se retrouve l'une au moins des trois espèces à savoir : A. africana, $K$. grandifoliola et $K$. senegalensis. Ainsi, quatre plantations d'enrichissement respectivement de 17, 18, 19 et 20 ans d'âge ont été identifiées avec des écartements variables $(2 \mathrm{~m} \mathrm{x} 2 \mathrm{~m} ; 2 \mathrm{~m} \mathrm{x} 4 \mathrm{~m}$ et $4 \mathrm{~m} \mathrm{x} 4$ $\mathrm{m})$.

\section{Dispositif d'inventaire et collecte des données}

Vingt quatre placeaux carrés de $30 \mathrm{mx}$ $30 \mathrm{~m}$, distants l'un de l'autre de $100 \mathrm{~m}$ au moins ont été installés au sein de chacune des plantations d'enrichissement considérées. Respectivement 6, 6, 7 et 5 placeaux sont installés dans les plantations d'enrichissement de $17,18,19$ et 20 ans d'âge, soit un total de 24 placeaux.

L'inventaire a consisté au recensement, au marquage systématique dans chacun des placeaux et à la mesure du diamètre de tous les sujets de diamètre supérieur ou égal à 10 $\mathrm{cm}$ à $1,30 \mathrm{~m}$ au-dessus du sol (dbh $\geq 10 \mathrm{~cm})$. De plus, la hauteur totale de tous les sujets de $\mathrm{dbh} \geq 10 \mathrm{~cm}$ de chacune des trois espèces cibles a été mesurée.

\section{Traitement des données \\ Paramètres structuraux de végétation}

Pour la caractérisation de la végétation, les paramètres structuraux ci-dessous ont été déterminés pour toutes les plantations d'enrichissement identifiées.

La richesse spécifique $S$, en espèces: elle représente le nombre total d'espèces présentes dans un milieu donné. 
L'indice de diversité de Shannon ( $H$, en bits):

$H=-\sum_{i=1}^{s} \frac{n_{i}}{n} \log \quad 2 \frac{n_{i}}{n}$

$n_{i}=$ nombre d'individus de l'espèce $i ; n=$ nombre total d'individus inventoriés dans les placeaux. Cet indice est maximal quand tous les individus sont répartis de façon équitable sur toutes les espèces.

L'équitabilité de Pielou ( $E q$ ) est une mesure de la régularité dans la distribution des individus de chaque espèce dans le peuplement:

$E_{q}=\frac{H}{H_{\max }}$ avec $H_{\max }=\log _{2} S$

$H_{\text {max }}=$ indice de diversité maximal théorique de Shannon lié au peuplement. L'indice $E q$ varie de 0 à 1 ; il est maximal quand les espèces ont des abondances identiques dans le peuplement et minimal quand un petit groupe d'espèces domine tout le peuplement.

La densité du peuplement ( $N$, en arbres/ha) est le nombre moyen d'arbres sur pied ramené à l'hectare, calculé par la formule: $N=\frac{n}{s}$

$n=$ nombre total d'arbres du placeau de superficie $S$ ( $S=0,09$ ha) ; ce paramètre a été calculé pour chaque type de plantation d'enrichissement.

Le diamètre de l'arbre de surface terrière moyenne ( $D g$, en $\mathrm{cm})$, obtenu par la relation:

$D_{g}=\sqrt{\frac{1}{n} \sum_{i=1}^{n} d_{i}^{2}}$

$n=$ nombre d'arbres du placeau et $d_{i}$, le diamètre $(\mathrm{cm})$ de l'arbre $i$.

La surface terrière ( $G$, en $\mathrm{m}^{2} /$ ha) est la somme des surfaces des sections transversales à $1,30 \mathrm{~m}$ du sol de tous les arbres, ramenée à l'hectare:

$$
G=\frac{\pi}{4 S} \sum_{i=1}^{n} 0,0001 d_{i}^{2}
$$

$d_{i}=$ diamètre (en $\mathrm{m}$ ) de l'arbre $i$ du placeau considéré et $S=$ surface du placeau en ha ( $S$ = 0,09 ha).

La contribution en surface terrière ( $C s$, en pour cent) est définie comme étant la part de chaque espèce cible dans la surface terrière de l'ensemble des arbres du placeau :

$C s=100 \frac{G p}{G} ;$

$G_{p}=$ surface terrière des arbres de $A$. africana, K. grandifoliola ou K. senegalensis et

$G$ =surface terrière de l'ensemble des arbres du placeau.

La hauteur moyenne de Lorey $\left(H_{L}\right.$, en m) est la hauteur moyenne des arbres, pondérée par leur surface terrière (Philip, 1998):

$H_{L}=\frac{\sum_{i=1}^{n} g_{i} h_{i}}{\sum_{i=1}^{n} g_{i}}$ avec $g_{i}=\frac{\pi}{4} d_{i}^{2}$

$g_{i}$ et $h_{i}$ étant respectivement la surface terrière et la hauteur totale de l'arbre $i$.

L'indice de valeur d'importance des espèces (IVI ), mesure le niveau d'importance des espèces dans le peuplement et permet d'identifier les espèces compétitrices potentielles. Il a été déterminé pour chaque espèce par la relation :

$I V I_{\alpha}=R D_{\alpha}+R F_{\alpha}+R C_{\alpha}$

$R D_{\alpha}=n_{\alpha} / \sum_{\alpha=1}^{p} \sum_{i=1}^{k} n_{(\alpha) i} \quad$ est la dominance de chaque espèce $\alpha ; n_{(\alpha) i}$ le nombre total d'individus de l'espèce $\alpha$ enregistré dans le placeau $i ; k$ est le nombre total de placeaux et $p$ le nombre total d'espèces dénombrées; $R F_{\alpha}=f_{\alpha} / \sum_{\alpha=1}^{p} f_{\alpha}$ avec $f_{\alpha}=j_{\alpha} / k$; $R F_{\alpha}$ est la fréquence de chaque espèce $\alpha$; $f_{\alpha}$ la fréquence de chaque espèce $\alpha$; $j_{\alpha}$ étant le nombre de placeaux où l'espèce 
$\alpha$ est retrouvée et $k$ le nombre total de placeaux; $\quad R C_{\alpha}=C_{\alpha} / \sum_{\alpha=1}^{p} \sum_{i=1}^{k} C_{(\alpha) i}$ avec $C_{(\alpha) i}=\left(A_{(\alpha) i} N_{(\alpha) i}\right) / n_{(\alpha) i} ; \quad R C_{\alpha}$ étant le recouvrement de chaque espèce $\alpha$; $A_{(\alpha) i}, \quad N_{(\alpha) i}$ et $n_{(\alpha) i}$ représentent respectivement la surface terrière $\left(\mathrm{m}^{2}\right)$, la densité des arbres (arbres $/ \mathrm{m}^{2}$ ) et le nombre total d'arbres de l'espèce $\alpha$, enregistrés dans le placeau $i$. L'indice de valeur d'importance des espèces varie de 0 à 3 . Plus sa valeur est élevée pour une espèce donnée, plus l'espèce est importante.

Les paramètres dendrométriques $N$, $G, D_{g}$ ont été calculés pour le peuplement arborescent des plantations d'enrichissement suivant l'âge et l'écartement. En effet, trois écartements ont été adoptés à savoir $2 \mathrm{~m}$ x 2 $\mathrm{m} ; 2 \mathrm{~m} \mathrm{x} 4 \mathrm{~m}$ et $4 \mathrm{~m} \mathrm{x} 4 \mathrm{~m}$. Par ailleurs, les paramètres $H_{L}, \quad C s$ et IVI ont été déterminés pour les populations d'espèces introduites (A. africana, K. grandifoliola et $K$. senegalensis) présentes au sein de ces plantations d'enrichissement.

Les populations des trois espèces cibles ont été comparées par le biais d'une analyse de la covariance au regard des différents écartements identifiés en considérant l'âge comme covariable sur la base des paramètres dendrométriques. La même analyse a été réalisée en prenant l'âge des plantations d'enrichissement comme facteur et l'écartement comme covariable. Le choix de ce test est dû aux influences probables de ces deux facteurs l'un sur l'autre. En outre, le test non paramétrique de Kruskal-Wallis a été effectué pour comparer les plantations d'enrichissement et les populations des trois espèces cibles sur la base des densités sur pieds.

\section{Structures en diamètre des plantations d'enrichissement}

Les individus de toutes les espèces ont été répartis en des classes de diamètre d'amplitude $5 \mathrm{~cm}$ et les densités (en arbres/ha) ont été calculées par classe de diamètre pour l'établissement des structures en diamètre des plantations d'enrichissement. En outre, les individus de A. africana, K. grandifoliola et $K$. senegalensis ont été répartis en des classes de diamètre d'amplitude $2 \mathrm{~cm}$ et les densités d'arbres par classe de diamètre ont été déterminées et représentées sous forme d'histogrammes.

Le mode de distribution des classes de diamètre est déterminé au moyen du coefficient d'asymétrie, $\beta_{1}$ qui mesure les proportions relatives des petites tiges par rapport aux grosses d'une population donnée et est défini comme suit (Weiner, 1990; Duncan, 1995):

$\beta_{1}=m_{3} /\left(m_{2}\right)^{3 / 2}$

L'asymétrie est positive ou négative, selon que $\mu_{3}$ est positif, indiquant que le maximum des observations est décentré vers la gauche; ou négatif, indiquant que le maximum des observations est décentré vers la droite.

Courbes de croissance en hauteur des sujets de A. africana, K. grandifoliola et $K$. senegalensis

Cinq modèles ont été testés à partir de 60, 152 et 256 couples d'observations (diamètre, hauteur totale) respectivement pour A. africana, $K$. grandifoliola et $K$. senegalensis. Il s'agit des modèles linéaires simples $(H=a+b D+\varepsilon)$ de Trorey $\left(H=a+b D+c D^{2}+\varepsilon\right)$, de Hendricksen $(H=a+b L n D+\varepsilon)$, de Stoffels et Van Soest $(\operatorname{LnH}=a+b \operatorname{Ln} D+\varepsilon)$ et de Avery et Burkhart $(\operatorname{LnH}=a+b / D+\varepsilon)$. Dans ces expressions, $D$ et $H$ sont respectivement le diamètre à $1,3 \mathrm{~m}$ du sol et la hauteur totale des arbres tandis que $a, b$ et $c$ sont les paramètres de régression des modèles à estimer, $\varepsilon$ le terme d'erreur et $\mathrm{Ln}=$ Logarithme naturel. 
RESULTATS

Caractérisation des plantations
d'enrichissement
Caractéristiques écologiques
espèces ligneuses dans les plantations est de 29 espèces avec un indice de diversité de Shannon égal à 3,4 bits qui traduit l'inégale répartition des individus des espèces présentes. On note l'abondance, dans l'ordre de rang décroissant, d'un groupe d'espèces (équitabilité de Piélou, $E q=0,7$ ) à savoir, T. superba, K. senegalensis, $H$. grandis, $C$. pentandra, K. grandifoliola, A. leiocarpa, $H$. floribunda, A. africana et D. mespiliformis. Les indices de valeur d'importance (IVI) varient entre 0,12 et 0,60 pour les huit espèces les plus importantes sur les 29 répertoriées. L'espèce la plus influente est $C$. pentandra tandis que $H$. floribunda est l'espèce la moins influente (Tableau 1).

\section{Caractéristiques dendrométriques}

Les moyennes et écart-types des paramètres dendrométriques pour les plantations d'enrichissement (Tableau 2) révèlent globalement une différence significative suivant l'âge de plantation des essences introduites. Les plantations d'enrichissement de 20 ans d'âge présentent des caractéristiques dendrométriques significativement différentes de celles des autres plantations avec notamment $26,6 \mathrm{~cm}$ et $37,3 \mathrm{~m}^{2} /$ ha respectivement pour le diamètre moyen des arbres et la surface terrière. Par ailleurs, en considérant les écartements de plantation, on note du Tableau 2 que seul le diamètre moyen varie significativement suivant l'écartement (prob. = 0,004). Le diamètre moyen le plus élevé $(26,1 \mathrm{~cm})$ est observé pour l'écartement $4 \mathrm{~m} \mathrm{x} 4 \mathrm{~m}$ tandis que la plus faible valeur moyenne $(21,1 \mathrm{~m})$ est notée pour l'écartement de $2 \mathrm{~m} \mathrm{x} 2 \mathrm{~m}$.

La densité moyenne d'arbres est estimée à 706 tiges/ha pour l'ensemble des plantations d'enrichissement avec un diamètre moyen et une surface terrière moyenne respectivement de $22,5 \mathrm{~cm}$ et $28 \mathrm{~m}^{2} /$ ha.

Par ailleurs, les caractéristiques moyennes des essences introduites dans ces plantations d'enrichissement (Tableau 3) révèlent une différence très significative entre les espèces, seulement pour la hauteur moyenne. Les arbres de A. africana présentent la hauteur moyenne la plus élevée $(13,9 \mathrm{~m})$ tandis que les sujets de $K$. senegalensis ont la hauteur moyenne la plus faible $(9,7 \mathrm{~m})$. Les autres paramètres dendrométriques présentent des différences non significatives suivant les essences forestières. On note une forte variabilité de ces paramètres dendrométriques des essences d'une placette d'inventaire à une autre au sein de la même plantation.

\section{Structures en diamètre des plantations d'enrichissement}

Les structures en diamètre des arbres des plantations d'enrichissement présentées à la Figure 1, montrent, pour les trois écartements $(2 \mathrm{~m} \times 2 \mathrm{~m}, 2 \mathrm{~m} \times 4 \mathrm{~m}$ et $4 \mathrm{~m} \times 4$ $\mathrm{m})$, une tendance fortement régressive d'allure quasi 'J renversé" caractéristique des peuplements multispécifiques ou inéquiennes avec une dominance des individus de petits diamètres. Les sujets ayant un diamètre compris entre 10 et $20 \mathrm{~cm}$ sont les plus représentés à l'hectare.

Par ailleurs, les structures en diamètres des essences forestières introduites présentent des coefficients d'asymétrie positifs (Tableau 4), ce qui traduit une allure dissymétrique de ces structures, conséquence d'une forte compétition entre les sujets des plantations d'enrichissement quel que soit l'écartement. Toutefois, les petits écartements induisent une compétition plus intense comparativement aux écartements plus grands.

\section{Croissance en hauteur des essences introduites}

La courbe de croissance en hauteur des essences forestières introduites dans les plantations révèle une allure logarithmique pour les trois espèces. Seul le modèle de Hendricksen a été retenu pour les espèces au regard du coefficient de détermination $\left(R^{\mathbf{2}}\right)$ relativement élevé avec une normalité et une homogénéité des résidus comparativement aux autres modèles. Ce modèle est globalement hautement significatif pour les trois espèces (prob. $=0,000$ ). Pour chacune d'elles, 50\% environ de la variabilité des hauteurs totales des sujets est expliquée par les variations de leurs diamètres. 
Tableau 1 : Indices de valeur d'importance (IVI) des espèces ligneuses dans les plantations d'enrichissement.

\begin{tabular}{lcc}
\hline Rang décroissant & Espèces ligneuses & IVI \\
\hline 1 & Ceiba pentandra & 0,60 \\
2 & Terminalia superba & 0,55 \\
3 & Khaya senegalensis & 0,33 \\
4 & Holoptelea grandis & 0,26 \\
5 & Khaya grandifoliola & 0,24 \\
6 & Anogeisus leiocarpa & 0,22 \\
7 & Afzelia africana & 0,13 \\
8 & Holarrhena floribunda & 0,12 \\
\hline
\end{tabular}

Tableau 2: Caractéristiques dendrométriques des plantations d'enrichissement suivant l'âge et l'écartement : valeurs moyennes (m) et coefficients de variation (cv, en \%).

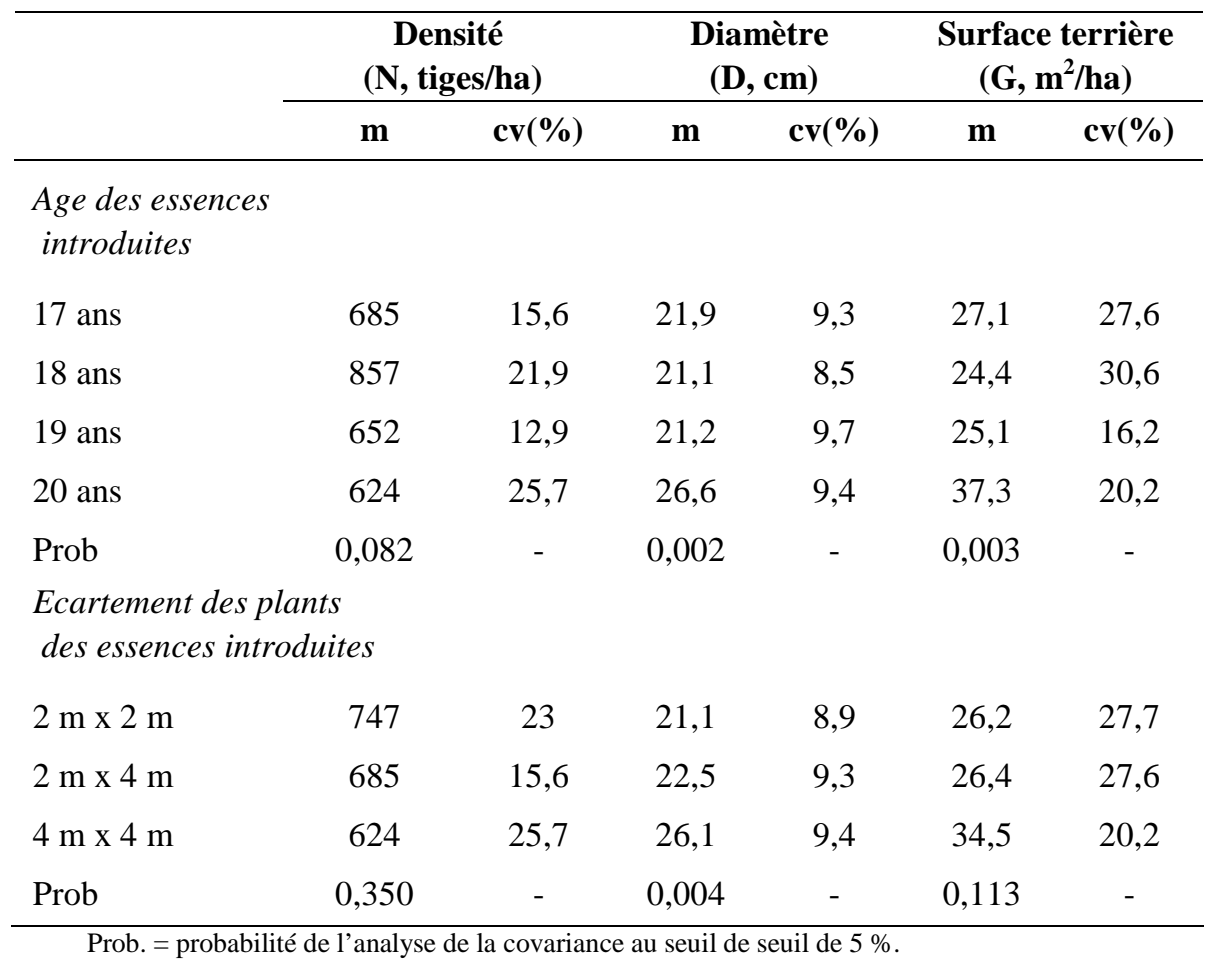




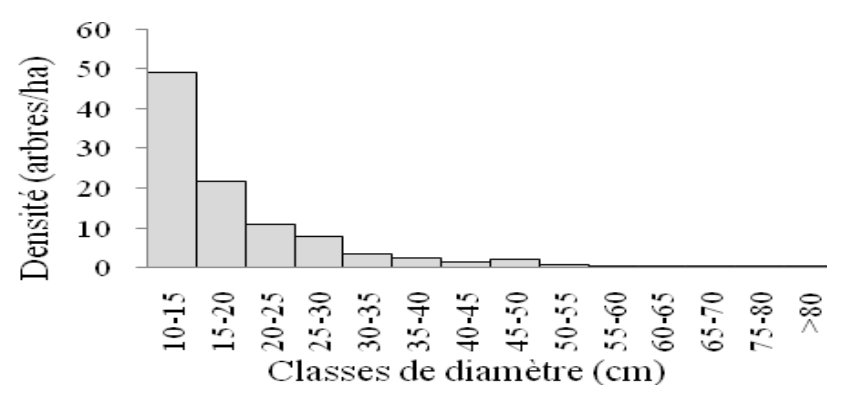

a) Ecartement de $2 \mathrm{~m} \times 4 \mathrm{~m}$

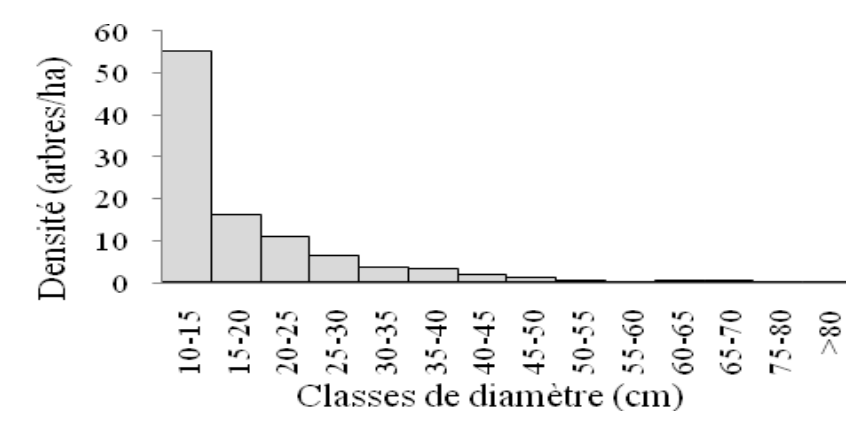

b) Ecartement de $2 \mathrm{~m} \times 2 \mathrm{~m}$

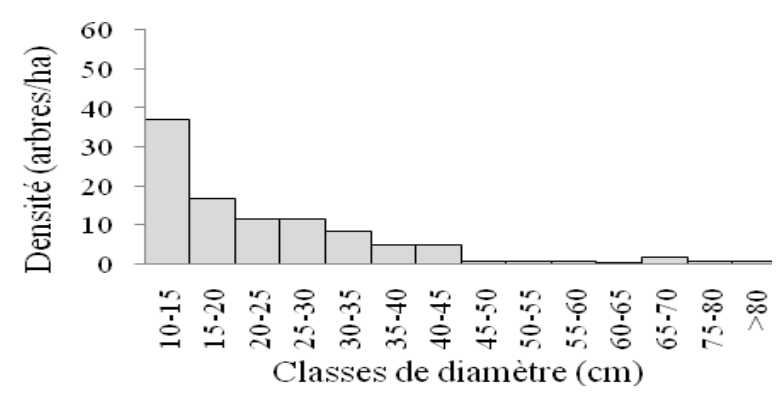

c) Ecartement de $4 \mathrm{~m} \times 4 \mathrm{~m}$

Figure 1 : Structures en diamètre des plantations d'enrichissement.

Tableau 3 : Paramètres dendrométriques des populations des essences introduites: moyennes $(\mathrm{m})$ et coefficients de variation (cv).

\begin{tabular}{|c|c|c|c|c|c|c|c|}
\hline \multirow[b]{2}{*}{ Paramètres } & \multicolumn{2}{|c|}{ A. africana } & \multicolumn{2}{|c|}{ K. grandifoliola } & \multicolumn{2}{|c|}{ K. senegalensis } & \multirow[t]{2}{*}{ Prob } \\
\hline & $\mathbf{m}$ & cv & $\mathbf{m}$ & cv $(\%)$ & $\mathbf{m}$ & $\operatorname{cv}(\%)$ & \\
\hline Densité (N, tiges/ha) & 65 & 57,5 & 90 & 96,9 & 136 & 87,5 & 0,115 \\
\hline Diamètre (D, cm) & 14,4 & 76,6 & 14,1 & 29,7 & 13,9 & 21,5 & 0,641 \\
\hline Surface terrière $\left(\mathrm{G}, \mathrm{m}^{2} / \mathrm{ha}\right)$ & 1,6 & 146,0 & 2,2 & 117,4 & 2,1 & 82,0 & 0,683 \\
\hline Hauteur $\left(\mathrm{H}_{\mathrm{L}}, \mathrm{m}\right)$ & 13,9 & 30,4 & 11,2 & 26,4 & 9,7 & 14,3 & 0,001 \\
\hline $\begin{array}{l}\text { Contribution à la surface } \\
\text { terrière (Cs, \%) }\end{array}$ & 5,7 & 140,3 & 7,9 & 116,5 & 7,5 & 77,5 & 0,812 \\
\hline
\end{tabular}


Tableau 4 : Paramètres d'asymétrie $\left(\beta_{1}\right)$ liés à la distribution des trois espèces en fonction des écartements des peuplements enrichis.

\begin{tabular}{lccc}
\hline Ecartements & A. africana & K. grandifoliola & K. senegalensis \\
\hline 2 m x 2 m & 1,1 & 1,9 & 1,5 \\
2 m x 4 m & - & 0,96 & 1,8 \\
4 m x 4 m & 0,99 & 0,9 & 0,9 \\
\hline
\end{tabular}

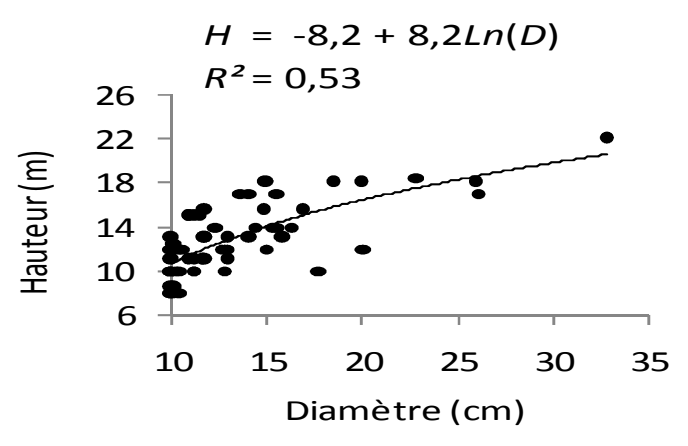

a) A. africana

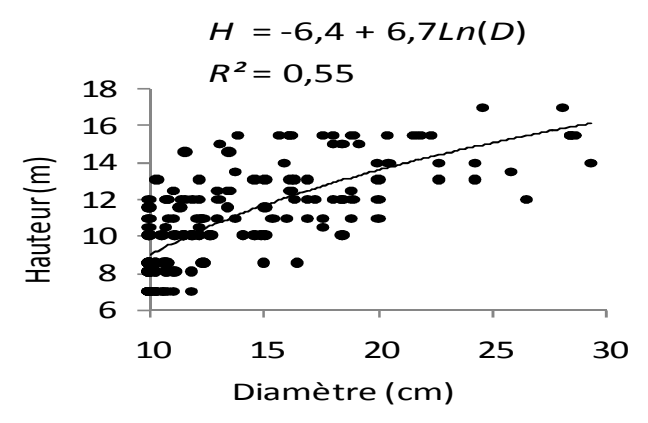

b) K. grandifoliola

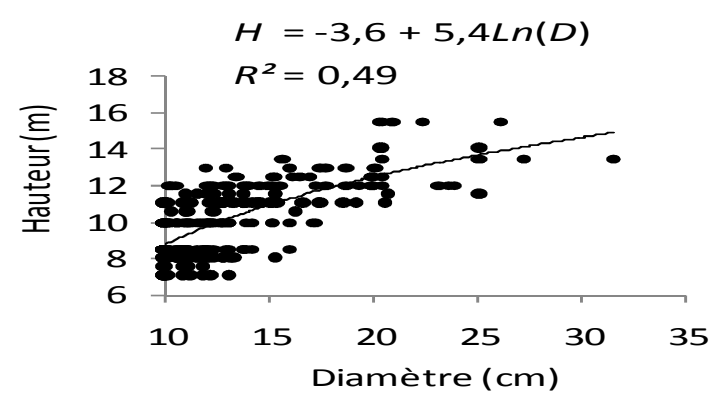

c) K. senegalensis

Figure 2 : Courbes de croissance en hauteur des sujets des trois espèces. 


\section{DISCUSSION}

Richesse floristique des stations et caractéristiques dendrométriques des plantations d'enrichissement

La perturbation de l'équilibre de la forêt naturelle du Noyau Central de la forêt classée de la Lama, due à la forte dégradation de sa structure par endroits, conséquence des actions anthropiques, a conduit à un enrichissement des zones dégradées, avec une vingtaine d'essences autochtones dont $A$. africana, K. grandifoliola et $K$. senegalensis. Ce reboisement avait pour but de rétablir l'équilibre écologique du Noyau Central, dernier grand vestige de forêt dense naturelle au Bénin (Djodjouwin et Sinsin, 2007). Vingt ans après les premiers enrichissements effectués, les zones enrichies de cet écosystème présentent globalement une physionomie floristique proche de celle de la forêt dense typique non dégradée même si des trouées y sont toujours observées par endroits. En effet, la richesse spécifique ligneuse obtenue (29 espèces) dans les plantations d'enrichissement est pratiquement la même que celle de la forêt dense non dégradée (31 espèces) (Bonou et al., 2009). Les compositions floristiques des deux milieux présentent toutefois des différences quelque peu importantes. Les espèces de la flore autochtone les plus abondantes dans les milieux enrichis sont Ceiba pentandra, Anogeissus leiocarpa et Holarrhena floribunda tandis que dans les formations non dégradées, Afzelia africana, Diospyros mespiliformis et Dialium guineense sont les plus abondantes. L'analyse croisée de la dominance et de l'importance des espèces introduites pour l'enrichissement révèle que $T$. superba est l'espèce la plus dominante et la deuxième espèce la plus importante après $C$. pentandra dans les peuplements enrichis. La prédominance des sujets de diamètre inférieur à $20 \mathrm{~cm}$ avec une structure en diamètre presque en 'J renversé" caractérise bien l'état jeune de ces peuplements enrichis mais aussi les effets probables de compétition inhibitrice de la croissance de la majorité des essences introduites.
En ce qui concerne les paramètres dendrométriques, la densité moyenne globale des plantations d'enrichissement (706 tiges/ha) est largement inférieure à la densité moyenne théorique estimée à partir des écartements considérés (1719 tiges/ha). La grande différence entre ces deux valeurs est la conséquence d'un taux de mortalité très élevé après la transplantation des plantules des essences introduites. Une telle situation s'explique par les compétitions intra et interspécifiques significatives qui règnent en permanence entre sujets voisins; les sujets introduits étant naturellement moins résistants dans le milieu naturel que ceux qui y sont originellement déjà présents. D'autre part, l'influence de la nature argileuse du sol (vertisol) du Noyau Central, notamment en saison pluvieuse n'est pas à négliger. Cette densité reste toutefois très significativement plus élevée que celle des espèces autochtones naturellement en peuplement dans le Noyau Central (144 tiges/ha) (Bonou et al., 2009), ce qui permet de prédire que cette diminution du nombre d'arbres plantés continuera très probablement dans le temps. Par ailleurs, il apparaît alors qu'un dispositif d'écartement 4 $\mathrm{m} \times 4 \mathrm{~m}$ permet une croissance en grosseur plus rapide des arbres et une compétition moins intense pour la lumière, en comparaison aux dispositifs de $2 \mathrm{~m} \mathrm{x} 4 \mathrm{~m}$ et $2 \mathrm{~m}$ x $2 \mathrm{~m}$ de densités théoriques respectives de 1250 et 2500 tiges/ha. Des résultats similaires ont été obtenus par Atindogbé (2004) qui a conclu que même si la distance entre plants peut varier selon les essences et les objectifs assignés à l'enrichissement, la fourchette communément admise va de 3 à $10 \mathrm{~m}$.

\section{Caractéristiques structurales des populations des essences introduites}

La contribution des trois espèces réunies à la densité globale des stations enrichies objet de la présente étude, est estimée à environ 40\%. Cependant, leur contribution totale en termes de surface terrière reste assez faible, environ $20 \%$ indiquant ainsi que les sujets des trois espèces sont encore de faibles diamètres malgré leur 
âge qui se situe entre 17 et 20 ans. Une telle croissance lente s'explique en partie par les effets de diverses compétitions que subissent les jeunes sujets introduits comparativement aux arbres déjà présents de façon naturelle dans le milieu. Ces trois essences, notamment $K$. senegalensis sont des espèces de pleine lumière et supportent mal la concurrence des autres arbres (Kelly et Cuny, 2000). A ces effets néfastes dus aux compétitions, est combiné le manque d'opérations sylvicoles appropriées, notamment les entretiens dans les premières années des stations d'implantations des individus introduits (Djodjouwin et Sinsin, 2007). Ces faibles valeurs sont en partie liées à la nature argileuse du sol (vertisol) de la forêt classée de la Lama qui ne permet pas un bon enracinement des arbres et une bonne exploitation des ressources du sol, surtout lorsque les jeunes arbres n'y sont pas régénérés naturellement. Les plus grandes valeurs de grosseur sont notées dans les plantations d'enrichissement où l'écartement est $4 \mathrm{~m} \mathrm{x} 4 \mathrm{~m}$ soit une densité théorique de 625 tiges/ha. Cet écartement semble donc celui favorable à une croissance en grosseur plus rapide des sujets introduits avec une compétition moins intense. Par ailleurs, la forme des structures en diamètre des essences s'écarte significativement de la structure gaussienne à laquelle l'on pourrait théoriquement s'attendre en présence de la distribution en diamètre de la population d'une espèce. De telles structures confirment les effets du phénomène de compétition entre sujets qui affectent fortement la croissance des individus d'espèces introduites pour des écartements plus réduits. Le stress environnemental dû aux intenses compétitions spécifiques entraîne en général, un défaut de croissance des petits sujets (Dhote, 1997). Ceci conduit assurément à une sélection progressive des sujets vigoureux et adaptés au milieu au détriment des sujets moins vigoureux et non adaptés qui, à la longue, disparaissent (Dhote, 1999). Dans les plantations d'enrichissement de la forêt classée de la Lama, la compétition a conduit en toute évidence à des structures dissymétriques avec une large représentativité des sujets de petits diamètres qui éprouvent des difficultés à croître normalement.

Quant aux courbes de croissance en hauteur, les corrélations relativement fortes entre les valeurs de diamètres et de hauteurs sont dues surtout au jeune âge des individus d'espèces introduites. En effet, la croissance en hauteur évolue dans le temps et à un certain âge du peuplement, les différences de croissance de hauteur entre les individus s'estompent de plus en plus (Philip, 1998; Rondeux, 1999).

\section{Conclusion}

La présente étude conduite dans les plantations d'enrichissement du Noyau Central de la forêt classée de la Lama, ciblée sur trois des espèces introduites à savoir, $A$. africana, $K$. grandifoliola et $K$. senegalensis a permis de mieux expliciter l'état actuel de leurs populations 20 ans après leur introduction dans cette station. Elles représentent $40 \%$ de la densité globale des espèces présentes dans les stations enrichies. Toutefois, leur surface terrière ne représente que $20 \%$ de la surface terrière de l'ensemble des arbres des peuplements enrichis, ce qui explique leur croissance en diamètre relativement lente et dénote que ces espèces introduites sont sujettes à une certaine compétition de la part des autres espèces présentes dans les peuplements enrichis. Au vu des caractéristiques écologiques et dendrométriques, des structures à tendance fortement régressive, il est nécessaire de réaliser des éclaircies destinées à favoriser la croissance et le dévéloppement des sujets d'avenir. Cette action pourra être désormais programmée dans les plans d'aménagement ou de gestion du Noyau Central de la forêt classée de la Lama avec une rubrique spécialement consacrée aux peuplements enrichis. Elle permettra de rétablir effectivement l'équilibre écologique de ces habitats qui jouent un rôle important dans l'équilibre global de la formation naturelle du Noyau Cenral. 


\section{REFERENCES}

Adomou AC, Akoegninou A, Sinsin B, De Foucault B, Van der Maesen LGJ. 2007. Biogeographical analysis of the vegetation in Benin. Acta Bot. Gallica, 154(2): 221-233.

Atindogbé G. 2004. Analyse de la structure spatiale des arbres: Application à l'enrichissement des savanes boisées de la zone soudanienne au Bénin. Thèse d'ingénieur Agronome, Université d'Abomey - Calavi, Bénin, P.97.

Barthod C. 1996. Sustainable management of temperate forests: the roots of the present international debate. Revue Forestière Française, 48: 13-22.

Bonou W, Glèlè Kakaï R, Assogbadjo EA, Fonton NH, Sinsin B. 2009. Characterisation of Afzelia africana $\mathrm{Sm}$. habitat in the Lama forest reserve of Benin. Forest Ecology and Management, 258: 1084-1092.

Djodjouwin L, Sinsin B. 2007. Expérience d'aménagement par enrichissement des formations forestières. In Actes du $3^{\text {ème }}$ séminaire international de RNSCC, Mayaka T, de Iongh H, Sinsin B (eds). Cotonou, Bénin ; 79 -96.

Dupuy B. 1989. Plaidoyer pour le reboisement dans les zones tropicales humides. Bois et Forêts des Tropiques, 221: 31-42.

Dupuy B. 1992. Les plantations à vocation de bois d'œuvre en forêt dense humide africaine. Bois et Forêts des Tropiques, 231: 5-15.

Duncan RP. 1995. A correction for including competitive asymmetry in measurements of local interference in plant populations. Oecologia, 103: 393-396.
Dhote JF. 1997. Effets des éclaircies sur le diamètre dominant dans des futaies régulières de hêtre ou de chêne sessile. Rev. For. Fr., 6: 557-578.

Dhote JF. 1999. Compétition entre classes sociales chez les chênes sessiles et le hêtre. Rev. For. Fr., 2: 309-324.

Ginisty C. 2008. Mélanger les peuplements pour une forêt plus riche, voire plus résistante au changement climatique. Le thème de recherche BIOFOR. Cemagref. $5 \mathrm{p}$.

Kelly BA, Cuny P. 2000. Plantations d'espèces forestières locales sur sols hydromorphes. Bilan d'une expérimentation sylvicole au sud du Mali. Rev.For.Fr., 5: 453-466.

Philip SM. 1998. Measuring Trees and Forests (Second Edition). 310 p.

Rondeux J. 1999. La Mesure des Peuplements Forestiers. Presses agronomiques de Gembloux : Belgique; p.522.

Sinsin B, Attignon SE, Lachat T, Peveling R, Nagel P. 2003. La forêt de Lama au Bénin: un écosystème menacé sous la loupe. Opuscula Biogeographica Basileensia, 3: 1-32.

Specht I. 2002. La orêt de la Lama, Bénin. SIG basé sur Landsat 7. Opusc. Biogeogr. Basileensa, 2 : 1-100.

Weiner J. 1990. Assymetric competition in plant population. Trends. Eco. Evol., 5: 360-364. 